\title{
Análisis crítico-filosófico de las potencialidades educativas de la enseñanza basada en competencias ${ }^{1}$
}

\section{Rodolfo Mauricio Bicocca-Gino}

Universidad de los Andes, Chile.

Conicyt - Fondecyt, Chile

mbicocca@uandes.cl

orcid.org/00oo-0003-1267-9033

\section{Resumen}

El actual paradigma hegemónico de la educación superior basado en el modelo por competencias es aún hoy motivo de discusión y debate en diversos círculos académicos. Este trabajo propone un análisis filosófico de la potencialidad formativa del enfoque con miras a desarrollar en el estudiante universitario disposiciones cognitivoreflexivas y ético-civicas. Se utiliza como método de investigación la hermenéutica documental. El escrito se articula en los siguientes puntos: 1) el desarrollo de disposiciones cognitivo-reflexivas y ético-cívicas en el estudiante de la educación superior; 2) el cambio de interés epistemológico en la educación superior; 3) la noción de competencia: antecedentes, supuestos y análisis del constructo; 4) críticas a la educación basada en competencias: un intento de sistematización; y, consideraciones finales. Se concluye que el enfoque carece de la potencialidad educativa requerida para generar en el estudiante las disposiciones para enfrentar las actuales sociedades complejas.

\section{Palabras clave}

Educación superior; pedagogía universitaria; enseñanza basada en competencias; disposiciones cognitivo-reflexivas; disposiciones ético-sociales (Fuente: Tesauro de la Unesco).

1 El presente trabajo fue posible gracias al financiamiento otorgado por Conicyt - Fondecyt, Chile, para el desarrollo del Proyecto Fondecyt Iniciación 11130078 (2013-2017) titulado: "El enfoque de capacidades (capabilities approach) de Amartya Sen y Martha Nussbaum. Un análisis fi losófi co critico y de sus potencialidades para la educación superior", actualmente en ejecución. Este artículo constituye un avance de resultados.

Recepción: 2015-10-21 | Envío a pares: 2016-01-10 | Aceptación por pares: 2017-01-30 | Aprobación: 2017-02-01

DOI: 10.5294/edu.2017.20.2.6

Para citar este articulo / To reference this article / Para citar este artigo

Bicocca-Gino, R. M. (2017). Análisis crítico-filosófico de las potencialidades educativas de la enseñanza basada en competencias. Educación y Educadores, 20(2), 267-281. DOI: 10.5294/edu.2017.20.2.6 


\title{
A Critical-philosophical Analysis of the Educational Potential of Competency-based Education
}

\begin{abstract}
The current hegemonic paradigm of higher education based on the competency model continues to be a subject of discussion and debate in a number of academic circles. This work proposes a philosophical analysis of the educational potential of the approach, specifically with a view towards developing cognitive-reflexive and ethical-civic aptitudes or readiness among university students. Documentary hermeneutics is used as a research method. The work centers on several points; namely, 1) the development of cognitive-reflective and ethical-civic aptitudes among university students; 2) the change of epistemological interest in higher education; 3) the notion of competence: background information, assumptions and construct analysis; and 4) criticism of competency-based education: an attempt at systematization, plus final considerations. The conclusion is that the approach lacks the educational potential required to generate the aptitudes or readiness students need to face the complex societies in today's world.
\end{abstract}

\section{Keywords}

Higher education; university teaching; competency-based instruction; cognitivereflexive aptitudes; ethical-social aptitudes (Source: Unesco Thesaurus). 


\section{Análise crítico-filosófica das potencialidades educativas do ensino com base em competências}

Resumo

O paradigma atual hegemônico da educação superior no modelo por competências é ainda hoje motivo de discussão e debate em diversos círculos acadêmicos. Este trabalho propõe uma análise filosófica da potencialidade formativa do enfoque para desenvolver no estudante universitário disposições congnitivo-reflexivas e ético-cívicas. Utiliza-se como método de pesquisa a hermenêutica documental. $O$ trabalho articula-se nos seguintes pontos: 1) o desenvolvimento de disposições cognitivo-reflexivas e ético-civicas no estudante da educação superior; 2) a mudança de interesse epistemológico na educação superior; 3) a noção de competência: antecedentes, hipóteses e análises do modelo teórico; 4) críticas à educação com base em competências: uma tentativa de sistematização; considerações finais. Conclui-se que o enfoque carece da potencialidade educativa necessária para gerar no estudante as disposições para enfrentar as complexas sociedades atuais.

\section{Palavras-chave}

Educação superior; pedagogia universitária; ensino com base em competências; disposições cognitivo-reflexivas; disposições ético-sociais (Fonte: Tesauro da Unesco). 


\section{Introducción}

Hoy, las universidades demandan a sus estudiantes adquirir competencias operacionales, es decir, habilidades operativas eficaces, legítimas y necesarias para el ejercicio de su profesión. Este imperativo lo asumió la educación superior (ES) con Bolognia (1999) y su propuesta de una educación basada en competencias (EBC). Han pasado ya más de quince años desde la reforma universitaria y hoy, en diversos ámbitos científicos y académicos, surgen dudas sobre sus resultados formativos (Díaz, 2006; Martínez Cinca, 2011).

Así, las universidades son objeto de debate (Boni y Walker, 2013) porque se busca analizar en qué grado colaboran a que el estudiante universitario adquiera disposiciones ${ }^{2}$ cognitivo-reflexivas y ético-sociales requeridas para un adecuado desarrollo profesional y humano. En tal sentido, la literatura científica muestra la relación que hay entre pensamiento crítico y ejercicio de una ciudadanía comprometida (Nussbaum, 2010; Medina, 2012).

Este problema motiva diversos cuestionamientos, tales como: jes suficiente para el estudiante universitario con adquirir saberes operativos para enfrentar la actual sociedad compleja? Y ¿es posible mantener y consolidar una democracia saludable solo con conocimientos técnicos? Estas, entre otras, son las preguntas que surgen del examen crítico que hacen distintos investigadores sobre la potencialidad formativa de las competencias y sus supuestos filosóficos en la universidad (Agut y Lozano, 2008; Walker, 2010, 2012; Lozano, Boni, Peris y Hueso, 2012; Rodríguez y Diestro, 2014).

El presente trabajo intenta mostrar cómo la $E B C$ es ciertamente insuficiente para un ajustado

2 La noción de disposiciones es de reciente introducción en el ámbito de la educación y no tiene aún una común aceptación. De hecho, fue introducida en la educación en Estados Unidos en los años noventa, por la Interstate New Teacher Assessment and Support Consortium para reemplazar el constructo de las actitudes (Schussler et al., 2010). desarrollo humano y profesional del estudiante de la ES, tanto para enfrentar la actual sociedad compleja y global (Raley y Preyer, 2010) como para construir un proyecto de vida sustentable. El escrito se articula en los siguientes puntos: 1) metodología de investigación; 2) el necesario desarrollo de disposiciones cognitivo-reflexivas y ético-cívicas en el estudiante de la ES; 3) el cambio de interés epistemológico de la ES; 4) la noción de competencia, antecedentes, supuestos y análisis del constructo; 5) críticas a la educación basada en competencias: un intento de sistematización; y conclusiones.

\section{Metodología de investigación}

La metodología utilizada para el desarrollo de la presente investigación es eminentemente cualitativa. Se utilizó la hermenéutica documental, consistente en la lectura, el análisis y la interpretación crítica de las fuentes bibliográficas. Este examen se ejecuta a partir de lo que sostiene H. G. Gadamer (1994) cuando afirma que "el intérprete indaga en un texto lo que hay propiamente en él. [...] y en esa remisión a lo que hay en el texto, este aparece como el punto de referencia frente a la cuestionabilidad, arbitrariedad o pluralidad de posibilidades interpretativas que apuntan a él". Por esta razón, el principio de literalidad del texto es una clave fundamental para su adecuada interpretación (Bicocca, 2008). A partir de esta metodología se desarrolló la investigación procediendo a un análisis detallado de la literatura científica de los autores pertinentes, seguido de un examen crítico de los argumentos expuestos y su posterior discusión. Asimismo, es necesario señalar que es imposible agotar toda la literatura que hay sobre el problema que se aborda en este trabajo.

\section{El desarrollo de disposiciones reflexivas y ético-cívicas en el estudiante de la ES}

Las democracias actuales, más que en otras épocas, necesitan educar a sus miembros como ciudadanos libres, capaces de participar y deliberar en la toma de decisiones políticas (Santos, 2013; Pal- 
mero, Jiménez y Jiménez, 2015). El problema estriba en que la EBC, por su matriz económica, tiende a reducir la formación del estudiante a su empleabilidad y descuida otras disposiciones requeridas para enfrentar las actuales sociedades complejas. En consecuencia, importantes disposiciones cognitivoreflexivas y ético-cívicas del sujeto de la ES quedan sin un desarrollo adecuado.

Se entiende por sociedades complejas aquellas comunidades que se caracterizan por sus cambios vertiginosos, exceso de información, alta tecnologización, globalización, ausencia de límites, cosmopolitismo y diversidad ideológica, cultural y religiosa (Morrison, 2008). En estas sociedades es donde el estudiante universitario deberá desarrollarse profesional y humanamente. De allí el carácter insuficiente de prepararlo solo para el ejercicio de saberes instrumentales que no ofrecen valoraciones superiores más allá del éxito profesional, el rendimiento económico o el consumo.

Hay ciertas disposiciones consideradas cruciales para una auténtica ES, que son propias de una razón teórica y práctica. En este sentido, una ES saludable debe generar espacios curriculares para que los estudiantes puedan desarrollar una inteligencia deseosa, lo que implica la generación de disposiciones cognitivo-reflexivas y ético-cívicas.

La relevancia de dichas disposiciones frente a la actual sociedad compleja es clave, ya que se relaciona, por una parte, con el despliegue del proyecto de vida del estudiante, porque aquellas le permitirían discernir los bienes necesarios para una vida buena (Finnis, 2011). Y, por otra, paradógicamente, con su empleabilidad. En efecto, el egresado universitario, para ser atractivo a una organización, debe mostrar iniciativa, creatividad y capacidad para establecer relaciones entre el mundo de la comprensión y el de la acción.

Una EBC orientada prioritariamente a preparar para la empleabilidad no propicia disposiciones como el deseo de saber por sí mismo y de conocer más, incluidas aquí la cultura, las artes y las letras, porque estas implican un lujo inútil y no se orientan a la profesionalización (Nussbaum, 2010). Por el contrario, una ES para la vida no solo no descuidaría el desarrollo de una razón instrumental, sino que también contendría espacios para el cultivo de las otras dimensiones de la razón humana, como son las razones teórica y la práctica, en tanto parte crucial de la educación universitaria.

Asimismo, es necesario destacar que la importancia del cultivo de las otras dimensiones de la razón radica en las siguientes distinciones: a través de la razón teórica se investigan y explican las reales causas de los eventos; por medio de la razón práctica se determinan los fines en vistas del actuar; por medio de la razón instrumental o técnica, dados los fines, se buscan las mejores maneras o vías para alcanzar aquellos. La razón teórica tiene principalmente que ver con las preguntas a cerca del qué y el por qué, y le concierne el conocimiento; la razón práctica tiene que ver con las preguntas a cerca del qué hago y por qué; y la razón instrumental se cuestiona por cómo hacerlo (Crespo, 2012).

En tal sentido, se entiende la ES como una instancia más del cultivo de la humanidad (Nussbaum, 2005). Esto es, una instancia capaz de despertar y encender las disposiciones superiores del estudiante, dado que gran parte de la misión de la universidad radica en desarrollar el inmenso potencial de la vida intelectual, y no solo la praxis y la resolución de problemas. En este marco de ideas, tiene pleno sentido el deseo de un reencuentro y una rehabilitación del ocio como uno de los elementos fundamentales del diseño de las políticas educativas actuales (Palmero, Jiménez y Jiménez, 2015).

\section{El cambio de interés epistemológico en la ES}

El debate actual sobre la ES reside, primero, en identificar y justificar cuáles son los propósitos más valiosos que se deben promover en el esfuerzo 
educativo, y segundo, en articular dichas finalidades; estos ejes posibilitan establecer las políticas públicas sobre ES (Lozano, Boni, Peris y Hueso, 2012).

El problema es que los fines de la ES hoy se encuentran trastocados. Ya no son internos a la dinámica de una razón vital, guiada por la búsqueda de un conocimiento esclarecedor del mundo. Hoy conviven en la ES intereses epistemológicos diversos. Por una parte, se encuentran las demandas originarias de la universidad: la investigación científica, la transmisión de la ciencia y la formación cultural y humana de nivel superior (Medina Rubio, 2005). Y, por otra, las crecientes demandas sociales y técnicoeconómicas inclinadas a saberes útiles.

En tal dirección, la OCDE plantea que el objetivo principal de la ES es: "una vida exitosa y el buen funcionamiento de la sociedad" (Rychen y Salganik, 2006). Objetivos postulados desde el inicio de la Unión Europea: "convertirse en la economía más competitiva del mundo a través de la reevaluación del conocimiento" (Comisión Europea, 2003). En continuidad con esta lógica, el Espacio Europeo para la Educación Superior (EEES) postuló una EBC. El objetivo de implementar este enfoque fue que permitiría ser un buen predictor de la actuación del egresado en su futuro ámbito laboral (McClelland, 1993, 1998).

A pesar de este aparente éxito, los principios pedagógicos que animan tal paradigma son discutibles (Martínez Cinca, 2011). En efecto, hoy los centros de ES son conscientes de que deben justificar su presencia por su utilidad económica. En consecuencia, se dirigen a sus alumnos como consumidores que pueden elegir en un mercado educativo competitivo, donde la empleabilidad se ha tornado la preocupación educativa prioritaria.

Ahora bien, la ES no puede abocarse a formar exclusivamente en saberes técnico-operativos, conforme los dictámenes de una razón instrumental, sin caer en un reduccionismo educativo, ya que las circunstancias vitales del egresado universitario y la complejidad de las sociedades actuales superan los límites de la capacitación laboral. Por estas razones, quien esté a favor de una educación humanista no puede desconocer que el enriquecimiento personal es un objetivo crucial de esta actividad (Cejudo, 2006).

Sin desmerecer la necesidad de aprender competencias y ser competente, se considera que además de enseñar a trabajar, la ES debe enseñar a pensar y a tomar buenas decisiones. Aunque estas disposiciones quizás no se orienten a la producción, seguro son necesarias tanto para una vida digna (Pele, 2010) como para un florecimiento humano (Jones, Lewis y Reffitt, 2013).

\section{Las competencias: antecedentes, análisis del constructo y supuestos}

El concepto de competencia surge en el ámbito de la psicología con R. White (1959) y luego ingresa al mundo empresarial con D. McClelland (1973), ante la insatisfacción de las medidas unidimensionales que se utilizaban entonces para predecir el rendimiento de las personas en un puesto de trabajo. Este autor convirtió la noción de competencia en la variable que revolucionó la gestión de los recursos humanos (Delamare Le Deist y Winterton, 2005). Luego en los años noventa, la literatura sobre dirección estratégica de empresas fue dominada por el concepto de competencias nucleares (Cappelli y Crocker-Hefter, 1996; Scarborough, 1998).

Desde el mundo de la empresa el término se extrapoló a la educación a través de la lingüística de Chomsky (1965), quien formuló el concepto de competencia lingüistica. A partir de aquí se generalizó su empleo, aplicándose a diversos ámbitos del saber (Perrenoud, 2008). Entre los países pioneros que implementaron una EBC se encuentran Estados Unidos, donde surge el denominado movimiento de la competencia, en los años setenta (Grant, 1979). Posteriormente, será Inglaterra quien lo aplique, en los años noventa, a través de la National Council for Vocational Qualifications. Estos son los precedentes 
relevantes del cambio curricular de la ES que implementó la Unión Europa, y que se concretaron en el Proceso de Bolonia (1999). Así lo manifiesta la misma Declaración cuando afirma:

Debemos mirar, en particular, por el objetivo de incrementar la competitividad del sistema europeo de educación superior. La vitalidad y la eficiencia de cualquier civilización se pueden medir por el atractivo que su cultura ejerce sobre otros paises. Necesitamos asegurarnos de que el sistema europeo de educación superior adquiera un grado de atracción de alcance mundial (Declaración de Bolonia, 1999, p. 2).

La declaración se refiere, en distintas partes, a dicha noción, sobre la cual se construye la metodología de gestión por competencias (Alles, 2006, 2009). No obstante, parece no haber una definición unívoca de dicho término (Días, 2006). Cuando se investiga la noción resulta paradójico comprobar que es imposible identificar y atribuir una teoría coherente o arribar a una definición precisa de aquella (Dalamare y Winterton, 2005). Además, los autores reconocen que la cuestión semántica de las competencias pedagógicas es controvertida (Westera, 2001; González y Ortiz, 2008; Moreno, 2009).

Así, Norris argumenta que "la palabra competencia ha sido excedida por la necesidad de definir y operacionalizar conceptos, la práctica ha envuelto a la teoría en una confusión, y algo aparentemente simple devino en algo complicado" (Norris, 1991, p. 332). En idéntica dirección, J. Boon y $M$. van der Klink (2002), definen la competencia como un "concepto confuso, pero útil para superar el espacio entre educación y trabajo".

En suma, no se ha alcanzado una delimitación clara y unánime, lo que agrava las dificultades del enfoque para ser aplicado, teniendo esto consecuencias para los colectivos involucrados en el EEES (Dalamare y Winterton, 2005). Aún hoy resulta imposible llegar a una definición que reconcilie los significa- dos del término competencia (Agut y Lozano, 2008; Moreno, 2009), pues los enfoques y las tradiciones desde los cuales se estudia y aplica son diversos. A lo dicho se agrega la dificultad, no menor, de trabajar y evaluar un constructo psicológico compuesto por diversas variables (Agut y Grau, 2001; Bautista-Cerró, 2007).

En general, la literatura existente se refiere a la noción de competencia como capacidades, habilidades, destrezas, estrategias, entre otras. Se sostiene que las competencias hacen referencia tanto a la posibilidad de evaluar un comportamiento predictivo como a las características de la personalidad, devenidas en comportamientos, que generan un desempeño exitoso en un puesto de trabajo (Aristimuño, 2008; Mora, 2011).

Otro elemento sugerente de análisis lo constituye el supuesto teórico que sustenta este enfoque de la ES, que es la teoría económica del Capital humano, que surge de un paradigma estrictamente económico y economicista, con una visión instrumentalista de la educación. Los exponentes más destacados de este enfoque fueron Theodore Schultz y Gary Becker en la década de los años sesenta, autores cuyos trabajos hicieron que la educación ganara importancia al reconocerle su contribución al desarrollo económico (Schultz, 1963, 1971, 1981, 1989; Becker, 1973, 1993).

La teoría del Capital humano subraya la importancia universal de la educación, pero sin reconocer en esta un valor sustantivo, sino el derivado de su aporte al crecimiento económico. Es decir, se focaliza en las habilidades productivas de cada persona, medidas en términos de bienes y servicios que produce, y considerando el consumo como la meta última de la actividad económica (Chiappero-Martinetti y Sabadash, 2010).

En tal sentido, la teoría del Capital humano tiene una visión instrumentalista de la educación (Lozano, Boni, Peris y Hueso, 2012), en cuanto que es una 
herramienta efectiva para la capacitación laboral. En efecto, se asume que trabajadores mejor capacitados son más productivos y generan mayor riqueza (Schultz, 1989). De este modo, esta teoría es invocada como el modelo de educación más adecuado para mejorar la vida individual y social conforme las normas dadas por el mercado y su productividad (Walker, 2012).

Hoy, entonces, la ES tiende al desarrollo prioritario de la razón instrumental, con claros objetivos inmediatos puestos en la preparación profesional y el éxito laboral (Mora, 2011), y objetivos mediatos orientados al crecimiento económico y la generación de riqueza (Nussbaum, 2012).

\section{Críticas a la educación basada en competencias: un intento de sistematización}

Las críticas al enfoque por competencias se pueden sintetizar en los siguientes puntos: a) una lógica instrumentalista, porque las competencias son herramientas para alcanzar "el éxito profesional y el buen funcionamiento social" (Rychen y Salganik, 2006); b) una falta de valores éticos centrales explícitos para desarrollar (Crocker, 2008; Vaughan y Walker, 2012); y c) el utilitarismo y el mercantilismo como valores últimos que orientan la actividad educativa (Wolf, 2002; Mehrotra, 2005; Lanzi, 2007). A continuación se profundiza en estos puntos a fin de explicitar los problemas que ofrece el enfoque en orden al desarrollo profesional y humano del estudiante universitario.

\section{Las competencias y su lógica instrumentalista}

Los contenidos que utiliza el enfoque son claves para determinar su carácter formativo. En tal sentido, el concepto de conocimiento representa un desafío importante para la EBC (Barnett, 2001). Aquí cabe preguntar, ¿con qué tipos de saberes trabaja el enfoque? y ¿a qué finalidades se orientan los conocimientos que este brinda?
El proyecto DeSeCo (2005) señala que "una competencia es la habilidad para satisfacer con éxito exigencias complejas en un contexto determinado, mediante la movilización de prerrequisitos psicosociales que incluyen aspectos cognitivos y no cognitivos" (Rychen y Salganik, 2006, p. 74). Perrenoud define la competencia "como la forma de actuar eficaz en un tipo definido de situación, capacidad que se apoya en conocimientos pero que no se reduce a ellos" (2008, p. 7). Finalmente, para M. Alles las "competencias hacen referencia a las características de la personalidad, devenidas en comportamientos, que generan un desempeño exitoso en un puesto de trabajo" (2009, p. 18).

Las definiciones anteriores ofrecen ciertos elementos de análisis. El primero radica en la dificultad de pensar una acción que no se sustente en conocimientos, como si la eficacia de aquella no dependiese exclusivamente del saber y del saber hacer que posee el agente que la ejecuta (Martínez Cinca, 2011). En tal sentido, el enfoque por competencias no ofrece una solución satisfactoria a la relación entre pensamiento y acción (Barnett, 2001). En efecto, una consecuencia lógica de invertir la tradicional superioridad del pensamiento por sobre la acción es dejar a la acción libre, sin las ataduras del pensar, para ser atada por cualquier otro fin, como por ejemplo, el mercado (Martínez Cinca, 2011) o alguna ideología.

Segundo, las definiciones señalan una misma dirección: se busca un conocimiento útil, eficaz y operativo, que genere resultados exitosos. En otras palabras, la EBC como constructo psicológico (Perrenoud, 2008) se orienta a la adquisición de habilidades operativas. Y el conocimiento que requiere este tipo de destrezas es el técnico-instrumental, donde la razón opera prioritariamente de manera resolutiva o estratégica. En esta dirección es sugerente lo que señalaba $\mathrm{H}$. Arendt cuando sostenía que:

...los totalitarismos del siglo XX pueden explicarse en gran medida por el desencanto de la razón, porque en una atmósfera en la que el éxito 
económico y la eficacia de la técnica valen más que el pensamiento crítico, en una sociedad en la que se evaporan los valores morales y las verdades tradicionales son ridiculizadas, resulta más fácil aceptar el éxito como único patrón de medida de la acción humana (1974, p.416).

Tercero, la EBC, debido al tipo de saberes instrumentales que promueve, llena el currículo de los estudios superiores de saberes expertos de alta utilidad técnica, pero también de alta caducidad temporal, y de una escasa potencialidad formativa de la persona. En efecto, los estándares ocupacionales no son absolutos y el conocimiento experto es altamente provisional al estar sometidos a la dinámica de la tecnología y la economía. De este modo, las competencias que hoy son necesarias ya no lo serán en unos años (Gimeno, 2008). En otras palabras, se le ofrece al estudiante universitario como contenido formativo un set de habilidades y destrezas que con rapidez creciente perderán su valor de uso.

Aquí es necesario destacar que el egresado universitario, para aplicar una determinada habilidad, requiere ser capaz de leer, interpretar y comprender la gramática de la situación-problema que deberá enfrentar; es decir, requiere adquirir ciertas disposiciones cognitivas no operativas, como la reflexión, la comprensión, la captación de significados, entre otras, que le permitan descifrar lo complejo de una situación determinada para saber actuar frente a ella. Se trata de disposiciones no instrumentales y que son propias de otras dimensiones de la razón humana. Ahora bien, cabe preguntar, ¿los conocimientos con los que trabaja la EBC contienen el potencial formativo para generar en los estudiantes tales disposiciones intelectuales?

\section{Las competencias y su insuficiencia de valores éticos centrales}

Un segundo centro de críticas a la EBC se dirige a su supuesta neutralidad valorativa. El enfoque se centra en la adquisición de habilidades técnicas para un accionar eficiente, supuestamente libre de connotaciones éticas. Aquí es necesario aclarar que la práctica educativa está transida de valoraciones y aquel que busca educar nunca puede ser un agente neutro. El accionar eficiente en un puesto de trabajo siempre implica deliberaciones éticas, algo que muestra el ejercicio de toda profesión.

Íntimamente relacionado con lo antes señalado, surge el problema de la predictibilidad que debe caracterizar a las competencias. Se piensa que la predictibilidad garantiza y asegura el desempeño exitoso del egresado. Ante lo cual cabe preguntar ¿cómo una conducta puede ser predecible y libre a la vez? Si la competencia es un actuar predecible y se ordenan los aprendizajes para que así lo sea, y de esto depende su evaluación, el estudiante requiere generar ciertas conductas automáticas esperables. Pero ¿esa automatización del pensamiento y la conducta acaso no predisponen y condicionan el juicio autónomo y el actuar libre del sujeto?

La predictibilidad que busca la EBC es discutible porque una genuina educación busca despertar el deseo por conocer, desarrollar el juicio propio, promover la autonomía y el carácter moral del individuo. Si la universidad no es cuidadosa, la automatización del trabajo mental, al cambiar la naturaleza y el foco de la empresa intelectual, podría erosionar una de las bases de la cultura occidental: el deseo por comprender el mundo (Carr, 2014). En tal sentido, las competencias son comportamientos y habilidades para actuar de manera deseada y definida por otros. Y, en consecuencia, reducen la autenticidad de la acción humana (Taylor, 1991; Barnett, 2009).

Por otra parte, a partir del análisis de algunas investigaciones empíricas, se concluye que la ausencia de valores éticos centrales de la EBC parece ser ya un hecho indiscutible en la ES actual. Algunos estudios muestran que el profesorado universitario es ajeno a las preocupaciones de una sólida educación ético-ciudadana y tienen cierto recelo a la hora de plantear tal tipo de formación como inherente a 
este nivel. Entre otras razones, porque se piensa que un aprendizaje ético-cívico no es responsabilidad de dicho colectivo (Martínez y Tey, 2008).

Otras investigaciones advierten que se considera la formación ético-cívica del estudiante como parte de la ES. No obstante, si bien los profesores piensan que sus estudiantes valoran tal formación, igualmente creen que, en general, estos conciben la universidad más como un lugar donde obtener un título profesional que de desarrollo personal. En suma, el profesorado asume que los estudiantes tienen una visión utilitarista y mercantil de la universidad, por más que múltiples declaraciones oficiales coloquen los aprendizajes ético-cívicos como cruciales para este nivel educativo (Bara, Mellen Vinagre y Buxarrais Estrada, 2014).

\section{Las competencias y sus valoraciones últimas: el economicismo y el mercantilismo}

El progreso económico es hoy uno de los objetivos principales de la educación, sino el principal. Importantes organizaciones supranacionales, como la OCDE y el Banco Mundial, consideran la educación como la herramienta más valiosa de dicho crecimiento (Gilead, 2012). Esta visión es acorde a la mentada teoría del Capital Humano, desde la cual el EEES asignó nuevas misiones a la universidad del siglo XXI: "intervenir en los procesos de innovación del sistema productivo y de las relaciones de la vida social" (Medina, 2005).

Es necesario destacar aquí lo cuestionable de los valores y el sentido a los que la EBC orienta la ES. El primero es su significativa valoración económica de la educación. En efecto, los únicos beneficios que son considerados son los obtenidos en orden a la productividad (PBI per cápita) y son descartadas todas aquellas dimensiones no económicas tanto de la educación como de la vida humana.

En tal dirección, esta visión no puede explicar las conductas de aquellas personas que quieren invertir su tiempo estudiando cosas que no tienen un claro retorno económico, como por ejemplo, aquellas personas que se abocan al cultivo de las artes o las humanidades (Robeyns, 2006). Asimismo, esto explica la actual reducción creciente del currículum de los estudios superiores a conocimientos estrictamente profesionalizantes, ya que todo saber que no se oriente a aumentar la empleabilidad del estudiante y su capacidad de generar riqueza no tiene valor de inversión.

Otro inconveniente radica en la mentalidad mercantilista que genera esta concepción cuando sus principios impregnan la vida individual y social. Esta mentalidad conduce a los estudiantes a comparar, conforme el criterio de rentabilidad, los beneficios monetarios que obtendrían con la carrera elegida. Siguiendo la misma lógica, los padres inducen a sus hijos a elegir ciertas carreras en virtud de su posible retorno económico (Robeyns, 2006). Esto trae aparejado la abundantísima matrícula de alumnos orientados a ciertas profesiones —las más lucrativas- en desmedro de otras igualmente necesarias para el bien general de la sociedad.

A lo antes afirmado hay que agregar el énfasis que pone el enfoque en la individualidad del estudiante en detrimento de su dimensión sociocomunitaria. Esto conduce a aquel a establecer relaciones individualistas y competitivas, centradas más en sí mismo y sus intereses personales que en el bien general. Es aquí donde los valores sociales que aportan a construir una convivencia más equitativa, solidaria, respetuosa y empática desaparecen y dejan paso a un criterio único y central de medición de la acción humana, a saber: el rendimiento económico. En sentido contrario, Drew Faust (2009), presidenta de la Harvard University, señala que si el modelo de mercado no se ha transformado en la identidad fundamental que define la educación superior, la universidad puede ofrecer a las personas y a las sociedades una profundidad y una amplitud de visión que se encuentran ausentes en una actualidad inevitablemente miope. Los seres humanos necesitan sentido, comprensión y perspectiva, además de trabajo. 


\section{Conclusiones}

La EBC sigue siendo hoy un enfoque cuestionable por su fuerte matriz economicista, sus debilidades y contradicciones teóricas, su falta de acuerdo unánime, sus problemas de aplicación, su extrañeza al ámbito educativo y por su sometimiento de la universidad a los criterios del mercado. En suma, se trata de un enfoque reduccionista e instrumentalista del fenómeno educativo.

Es necesario replantearse si este enfoque ayuda a que el estudiante universitario pueda afrontar su vida profesional y personal en las actuales sociedades complejas, donde la diferencia entre el cultivo o no de disposiciones cognitivo-reflexivas y ético-sociales puede implicar la diferencia entre una vida lograda y otra alienada pero económicamente satisfecha.

Ante esto se sostiene que las personas y su desarrollo vital están por encima de la mera generación de bienes de consumo. La ES debe iluminar y desarrollar aquellas disposiciones superiores del estudiante, o, lo que es lo mismo, considerar y promover las diversas dimensiones de la racionalidad de los estudiante o aquellas no económicas de la vida del sujeto.

El grave problema de la racionalidad instrumental que hoy impregna la ES es su tendencia a la mecanización del pensamiento y de la acción humana, porque cuando el parámetro para juzgar el valor del actuar se reduce a su eficiencia, y no se examinan el por qué y el para qué del mismo, o sea el fin, sucede que el sujeto eficiente deja de pensar y de evaluar cuáles son los reales móviles de su obrar, ya que otros los definen por él.

En este sentido, el inconveniente de preparar solo sujetos competentes, en los que la educación de disposiciones cognitivo-reflexivas y ético-cívicas no es algo prioritario en el currículo, es tanto su incapacidad para la lectura crítica y actuación frente a situaciones nuevas como su alta disposición a ser funcionales a cualquier sistema de valores o ideología.

Ante tal situación, urge pensar en una propuesta superadora que busque preparar no solo sujetos competentes y económicamente exitosos, sino también personas completas y ciudadanos socialmente comprometidos. Esta empresa sería posible desde un enfoque curricular no reduccionista de la educación humana y que considere necesarios también los saberes no instrumentales, como son las humanidades y las artes. Es decir, aquellos conocimientos que permiten el despliegue adecuado de las diversas dimensiones de la racionalidad y de la existencia de una persona.

\section{Referencias}

Agut Nieto, S.y Grau, R. (2001). Una aproximación psicosocial al estudio de las competencias, Proyecto Social, 9, 1-7.

Agut Nieto, S.y Lozano, A. (2008). Las competencias a debate: su papel en el proceso de convergencia europea en educación superior. Revista de psicología generaly aplicada, 61 (1), 53-68.

Alles, M. (2006). Dirección estratégica de recursos humanos. Gestión por competencias. Buenos Aires: Granica.

Alles, M. (2009). Nuevo Enfoque. Diccionario de competencias. La trilogía: las 60 competencias más utilizadas. Buenos Aires: Granica. 
ISSN 0123-1294 | e-ISSN 2027-5358 | Educ.Educ. Vol. 20. No. 2 | Mayo-agosto de 2017 | pp. 267-281.

Universidad de La Sabana | Facultad de Educación

Arendt, H. (1974). Los orígenes del totalitarismo. Madrid: Taurus.

Aristimuño, A. (2008). Las competencias en la educación superior: ¿demonio u oportunidad? Osorno: Centro de Documentación Universitaria.

Bara, F. E., Mellen Vinagre, T. y Buxarrais Estrada, M. R. (2014). Concepciones del profesorado universitario sobre la formación ética y ciudadana en el Espacio Europeo de Educación Superior: un estudio de caso. Revista de universidad y sociedad del conocimiento, 11 (3), 22-32.

Barnett, R. (2001). Los límites de la competencia. Barcelona: Gedisa.

Barnett, R. (2009). Rethinking the University after Bologna. New Concepts and Practices beyon the Tradition and the Market. Antwerpen: Universitair Centrum Sint-Ignatius Antwerpen.

Bautista-Cerró Ruiz, M. J. (2007). El largo camino de las competencias. Diseño de perfiles y programas. Acción pedagógica, 16, 6-16.

Becker, G. (1973). Human Capital. New York: Bureau of Economic Research.

Becker, G. (1993). Human Capital: A Theoretical and Empirical Analysis, with Special Reference to Education. Chicago: Chicago University Press.

Bicocca, M. (2008). Elementos en la metodología de la investigación filosófica. Intus-Legere, 2 (1), 95-108.

Boni, A., Walker, M. (2013). Human Development and Capabilities. Re-imagining the University of the Twenty-First Century. London: Routledge.

Boon, J. y van der Klink, M. (2002). Competencies: the triumph of a fuzzy concept. Academy of Human Resource Development, Annual Conference, Honoluly, 1, 327-334.

Cappelli, P.y Crocker-Hefter, A. (1996). Distinctive human resources are firms' core competencies. Organisational Dynamics, 24 (3), 6-22.

Carr, N. (2014). Atrapados. Cómo las máquinas se apoderan de nuestras vidas. Madrid:Taurus.

Cejudo Córdoba, C. (2006). Desarrollo humano y capacidades. Aplicaciones de la teoría de las capacidades de Amartya Sen a la educación. Revista española de pedagogía, 234, 365-380.

Chiappero-Martinetti, E. y Sabadash, A. (2010). Human capital and human capabilities: towards a theoretical integration. Pavia: University of Pavia.

Chomsky, N. (1965). Aspects of Theory of Syntax. Cambridge: MIT Press.

Crespo, R. (2012). Theoretical and Practical Reason in Economics. London: Springer. 
Crocker, D. (2008). Ethics of Global Development: Agency, Capability, and Deliberative Democracy. Cambridge: Cambridge University Press.

Dalamare Le Deist, F.y Winterton, J. (2005). What is Competence? Human Resource Development International, $8(1), 27-46$.

Deseco Proyect (2005). The definition and selection of key competencies. Excutive Summary.

Díaz Barriga, A. (2006). El enfoque de competencias en la educación. ¿Una alternativa o un disfraz de cambio? Perfiles educativos, 111, 7-36.

Declaración de Bolonia. Recuperado de http://www.ond.vlaanderen.be/hogeronderwijs/bologna/documents/ MDC/BOLOGNA_DECLARATION1.pdf

Faust, C. D. (2009). The University's crisis of purpose. New York Times Book Review, 6 de septiembre.

Finnis, J. (2011). Natural Law and Natural Rights. Oxford: Oxford University Press.

Gadamer, H. G. (1994). Verdad y método. Salamanca: Sígueme.

Gilead, T. (2012). Education and the logic of economic progress. Journal of Philosophy of Education, 46 (1), 113-131.

Gimeno, J. (2008). Diez tesis sobre la aparente utilidad de las competencias en educación. En Gimeno, J. (ed.). Educar por competencias ¿Qué hay de nuevo? (pp. 15-58). Madrid: Morata.

Grant, G. (1979). On Competence: A Critical Analysis of Competence-Based Reforms in Higher Education. San Francisco: Jossey-Bass.

González y Ortiz, F.X., (2008). Reseña sobre Barnett, Ronald. Los límites de las competencias. Investigación bibliotecológica, 22 (46), 229-236.

Jones, M. L., Lewis, P. A. y Reffitt, K. E. (eds.) (2013). Toward Human Flourisihing. Character, Practical Wisdom, and Professional Formation. Georgia: Mercer University Press.

Lanzi, D. (2007). Capabilities, Human Capital and Education. The Journal of Socio-Economics, 36, 424-435.

Lozano, J. F., Boni, A., Peris, J. y Hueso, A. (2012). Competencies in higher education: a critical analysis from capability approach. Journal of Philosophy of Education, 46 (1), 132-147.

Martínez Cinca, C. D. (2011). Límites de la educación superior basada en competencias. UDUAL, 4 (9), 59-77.

Martínez Martín, M.y Tey Teijón, A. (2008). Aprendizaje ético en contextos virtuales en el EEES. Revista Electrónica Teoría de la Educación, 9 (1), 25-40.

McClelland, D. (1973). Testing for competencies rather than intelligence. American Psychologist, 28, 1-14. 
ISSN 0123-1294 | e-ISSN 2027-5358 | Educ.Educ. Vol. 20. No. 2 | Mayo-agosto de 2017 | pp. 267-281.

Universidad de La Sabana | Facultad de Educación

McClelland, D. (1993). Intelligence is not the best predictor of job performance. Current Directions in Psychological Science, 2 (1), 5-6.

McClelland, D. (1998). Identifying competencies with behavioral-event interviews. American Psychological Society, 9 (5), 331-339.

Medina Rubio, R. (2005). Misiones y funciones de la universidad en el espacio europeo de la educación superior. Revista Española de Pedagogía, 230, 17-42.

Medina Plana, R. (2012). Critical thinking inside law schools: An outline. Oñati Socio-Legal Series, 2 (5), 7-24.

Mehrotra, S. (2005). Human capital or human development? Search for a knowledge paradigm for education and development. Economic and Political Weekly, 40 (4), 300-306.

Moreno Olivos, T. (2009). Competencias en educación superior: un alto en el camino para revisar la ruta de viaje. Perfiles educativos, 124, 69-92.

Mora, J. G. (2011). Formando en competencias: ¿un nuevo paradigma? Londres: Institute of Education, University of London.

Morrison, K. (2008). Educational philosophy and the challenge of complexity theory. En Mason, M. (ed.). Complexity Theory and the Philosophy of Education (pp.16-31). Oxford:Wiley-Blackwell.

Norris, N. (1991). The trouble with competence. Cambridge Journal of Education, 21 (3), 1-11.

Nussbaum, M. (2005). El cultivo de la humanidad. Barcelona: Paidos.

Nussbaum, M. (2010). Sin fines de lucro. Buenos Aires: Katz.

Nussbaum, M. (2012). Crear capacidades. Barcelona: Paidos.

Pele, A. (2010). La dignidad humana. Madrid: Dykinson.

Palmero Cámara, C., Jiménez Martín, J. y Jiménez Eguizábal, A. (2015). Ocio, política y educación. Reflexiones y retos veinticinco siglos después de Aristóteles. Revista española de pedagogía, 260, 5-21.

Perrenoud, P. (2008). Construir competencias desde la escuela. Santiago de Chile: J. C. Sáez.

Raley, Y.y Preyer, G. (eds.) (2010). Philosophy of Education in the Era of Globalization. New York: Routledge.

Robeyns, I. (2006). Three models of education. Rights, capabilities and human capital. Theory and Research in Education, 4 (1), 69-84.

Rodriguez Bravoa, A. E. y Diestro Fernández, A. (2014). Limitaciones teóricas del enfoque de competencias en el análisis de relación entre educación y empleo en el contexto europeo. Social and Behavioral Sciences, 139, $72-78$. 
Ry chen, D. S. y Salganik, L. H. (2006). Las competencias clave para el bienestar personal, social y económico. Málaga: Ediciones Aljibe.

Santos Rego, M. A. (2013). Cosmopolitismo y educación. Aprender a trabajar en un mundo sin fronteras. Valencia: Brief.

Scarborough, H. (1998). Path(ological) dependency? Core competencies from an organisational perspective. British Journal of Management, 9, 219-232.

Schultz, T. W. (1963). The Economic Value of Education. New York: Columbia University Press.

Schultz, T. W. (1971). Investment in Human Capital: The Role of Education and of Research. New York: Free Press.

Schultz, T.W. (1981). Investing in people: The economic of population quality. Berkeley: University of California Press.

Schultz, T. W. (1989). Investments in people: Schooling in low income countries. Economics of Education Review, 8, 219-223.

Schussler, D. L., Stooksberry, L. M. y Bercaw, L. (2010). Undertanding teacher candidate dispositions: reflecting to build self-awareness. Journal of Teacher Education, 61 (4), 350-363.

Taylor, Ch. (1991). The Ethics of Authenticity. Cambridge: Harvard University Press.

Vaughan, R. y Walker, M. (2012). Capability, values and education policy. Journal of Human Development and Capabilities, 13 (3), 495-512.

Walker, M. (2010). A human development and capabilities 'prospective analysis' of global higher education policy. Journal of Education Policy, 25 (4), 485-501.

Walker, M. (2012). Universities and a human development ethics: A capabilities approach to curriculum. European Journal of Education, 47 (3), 448-461.

Westera, W. (2001). Competences in education: a confusion of tongues. Journal of Curriculum Studies, 33 (1),75-88.

White, R. (1959). Motivation reconsidered: the concept of competence. Psychological Review, 66, 279-333.

Wolf, A. (2002). Does Education Matter? Myths about Education and Economic Growth. London: Penguin. 